\title{
Valeurs et fonctions du costume pour le rôle de Lavinia dans Titus Andronicus : un costume-corps ou un corps-costume?
}

Isabelle Beucher

\section{OpenEdition}

\section{Journals}

Édition électronique

URL : http://journals.openedition.org/shakespeare/1450

DOI : 10.4000/shakespeare.1450

ISSN : 2271-6424

Éditeur

Société Française Shakespeare

Édition imprimée

Date de publication : 20 décembre 2008

Pagination : 1-15

ISBN : 2-9521475-5-8

Référence électronique

Isabelle Beucher, "Valeurs et fonctions du costume pour le rôle de Lavinia dans Titus Andronicus : un costume-corps ou un corps-costume? », Actes des congrès de la Société française Shakespeare [En ligne], 26 | 2008, mis en ligne le 20 décembre 2008, consulté le 21 décembre 2020. URL : http:// journals.openedition.org/shakespeare/1450; DOI : https://doi.org/10.4000/shakespeare.1450 


\section{Costume et déguisement dans le théâtre de Shakespeare et de ses contemporains}

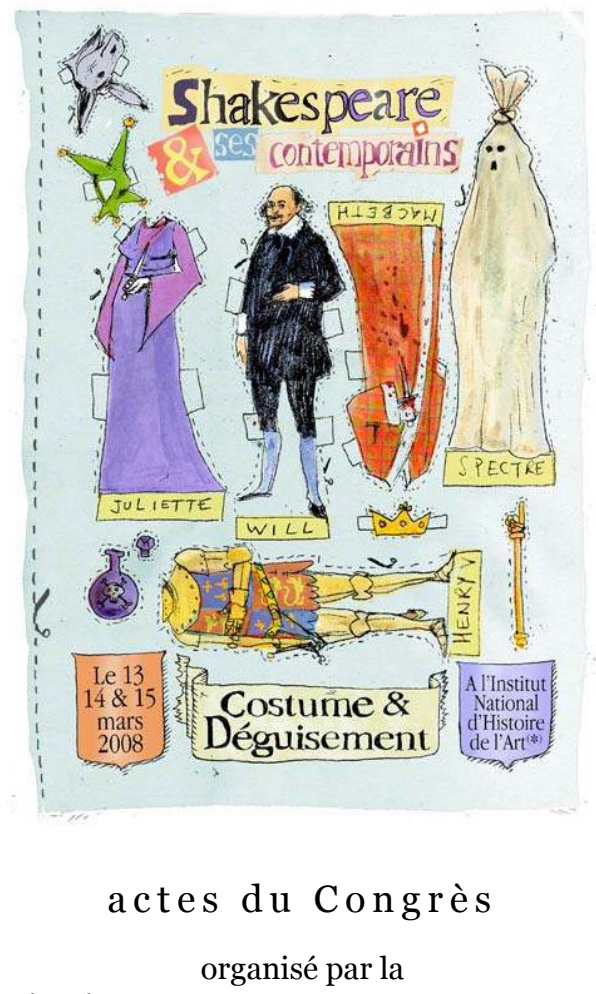

SOCIÉTÉ FRANÇAISE SHAKESPEARE

les 13,14 et 15 mars 2008

textes réunis par

Pierre KAPITANIAK

sous la direction de

Jean-Michel DÉPRATS 
COUVERTURE :

Edouard Lekston,

William en carton et ses habits à découper, 2008 edlek@free.fr

conception graphique et logo

Pierre Kapitaniak

\section{(C) 2008 Société Française Shakespeare}

Institut du Monde Anglophone

Université de Paris III - Sorbonne Nouvelle

5 rue de l'École de Médecine

75006 Paris

www.societefrancaiseshakespeare.org

Tous droits de traduction, de reproduction et d'adaptation réservés pour tous les pays 


\title{
VALEURS ET FONCTIONS DU COSTUME POUR LE RÔLE DE LAVINIA DANS TITUS ANDRONICUS : UN COSTUME-CORPS OU UN CORPS-COSTUME?
}

\author{
Isabelle BEUCHER
}

\begin{abstract}
Lavinia, dans Titus Andronicus, est un rôle problématique : le corps de la comédienne doit signifier les outrages les plus difficilement représentables. Le costume acquiert alors une fonction d'autant plus capitale : signifier le hors-scène, l'occulté. A travers l'étude de plusieurs mises en scène qui opèrent des choix radicalement différents de traitement du corps nous interrogerons la notion de costume ainsi que ses limites. Brook fait de Lavinia une figure allégorique de la souffrance. Le costume est une façade impénétrable, qui protège le corps réel mais réduit la fonction de l'acteur. Leret propose une version extrême de confusion du corps réel et du costume, exposant le sexe sanglant de son actrice. Le costume peut se faire corps, être à interpréter comme autant de signes de la souffrance du personnage. Le corps peut aussi se faire costume : il ne s'agit pas de le mutiler réellement mais de l'inscrire dans une sémiotique agressive.

In Titus Andronicus, Lavinia is a very problematic part: the actress's body must express ravages which are among the most difficult to represent. The role that the costume acquires then becomes all the more important: to signify what happened out of the stage, what has been concealed. Through the study of several productions which operate radically different approaches to the body, we will endeavour to analyse the notion of costume as well as its limits. Brook turns Lavinia into an allegorical figure of suffering. The costume is an impenetrable façade, which protects the actual body but reduces the actor's function. In Leret's production, the confusion between the actor's actual body and the costume is acute, and the actress's bleeding sex is exposed for all to see. Costumes can become bodies and be interpreted as sheer signs of the character's sufferings. The actor's bodies ca also become costumes: the aim is obviously not to actually mutilate them but to integrate them into an aggressive semiotic network.
\end{abstract}

la fin de l'acte v de Titus Andronicus, alors que pratiquement $\triangle$ tous les protagonistes se sont entretués, Lucius prend la parole 1 devant le peuple romain et justifie ses actes et ceux de son père. Cette tirade ne semble pas directement liée au sujet que je me suis proposé de traiter : le costume et le corps de Lavinia, mais elle est révélatrice du fonctionnement dramaturgique de la pièce et en cela elle peut être éclairante.

Eh bien, noble assistance, apprends

Que ce sont Chiron et le maudit Demetrius

Qui ont assassiné le frère de notre empereur,

Ce sont eux qui ont violé notre sœur :

Pour ces cruels forfaits, nos frères ont été décapités,

Les larmes de notre père méprisées, et lui-même bassement floué

De cette main loyale qui jusqu'au bout avait lutté pour Rome

Et expédié ses ennemis dans la tombe.

Moi-même enfin, inhumainement proscrit,

Les portes sur moi fermées, chassé en pleurs,

À devoir quémander secours chez les ennemis de Rome, 
Qui noyèrent leur hostilité dans mes larmes sincères

Et à bras ouverts m'accueillirent en ami.

Je suis cet expulsé, sachez-le,

Qui de mon sang ai assuré le succès de Rome,

Et, du sein de la patrie, ai détourné la pointe de l'ennemi,

Offrant comme fourreau à l'acier mon corps aventureux.

Hélas ! vous savez que je ne suis pas vantard, moi,

Mes cicatrices peuvent attester, muettes qu'elles sont,

L'exactitude de mon récit et sa véracité1.

Ce qui retient mon attention, ce sont surtout les trois derniers vers. Ils soulignent que les discours sont sujets à caution. Le peuple romain n'est pas obligé de croire les révélations que fait ici Lucius; en revanche ses blessures font office de preuve recevable. Dans la pièce, l'exhibition du corps souffrant ou plutôt ayant souffert atteste publiquement du discours. Si l'on ne demande pas finalement à Lucius de s'exécuter dans cette scène et d'exposer ses blessures, il n'en est pas de même de Lavinia, qui, à partir de la fin de l'acte II, alors qu'elle vient d'être violée et mutilée par les fils de Tamora, ne va cesser d'offrir à la vue des spectateurs son corps supplicié. Quand il évoque «ses cicatrices », Lucius semble paraphraser Lavinia, " muette qu'elle est » et dont le corps peut témoigner de « l'exactitude et de la véracité » de ce qu'elle a subi. Lavinia ne dispose en effet d'aucun autre moyen pour révéler ses souffrances. Elle n'est plus qu'une figure opaque et brute, un corps vivant qui ne produit plus de discours et auprès de qui les discours des membres masculins de sa famille se vident et semblent inopérants. Lavinia est un rôle sans doute difficile à tenir pour une comédienne, parce qu'elle est à la fois très présente sur scène mais réduite, à partir de l'acte II, au mieux à une pantomime. Comment, dans ce cas, dire l'outrage subi ? Certes, ce supplice est inscrit à l'intérieur d'une intrigue dont on suit le développement. D'autres protagonistes nous en font part : Aaron, à plusieurs reprises, fait un récit programmatique du sort qui est réservé à Lavinia. Il convoque le mythe de Philomèle et propose à Démétrius et Chiron d'entrer en concurrence avec Procné et d'être plus terrible que lui encore. Il crée ainsi une attente, une appréhension chez le spectateur, qui ne va pas directement assister au viol et aux mutilations, mais qui peut facilement se les figurer, et laisser à son imagination le soin de

${ }^{1}$ Il s'agit ici de la traduction de Jean-Pierre Richard pour l'édition de La Pléiade, dirigée par Jean-Michel Déprats, 2002. v.iii.95-114. 
concevoir le pire. Contrairement à ce qui est souvent d'usage dans la tragédie, le récit de l'acte sanglant n'a pas lieu après sa réalisation mais avant. Aucun discours efficace ne raconte véritablement ce que Lavinia a subi, comment l'acte barbare pensé et programmé a été effectivement réalisé. Le long monologue de Marcus, que d'aucuns ont jugé si problématique ${ }^{2}$, empreint de pétrarquisme et d'allusions littéraires, n'est finalement pas d'un grand secours. C'est donc à une confrontation directe, sans la médiation réelle d'un discours, que le spectateur est confronté au corps de Lavinia. Dès lors Shakespeare laisse le champ libre au théâtre à proprement parler. C'est à la mise en scène de suppléer ce vide laissé volontairement par l'auteur, de donner à voir ce qui est inscrit dans l'intrigue et que seule une didascalie éclaire : « entre Lavinia violée, la langue et les mains coupées » (II.iv.).

De quels moyens dispose le théâtre pour figurer le viol et les mutilations?

C'est ici que le costume de théâtre acquiert, me semble-t-il, une fonction déterminante. Il se substitue en effet au discours, et le moindre élément qui le constitue va être un signe tangible du viol et des mutilations. La question qui se pose alors est celle du degré d'implication du corps réel du comédien dans la figuration de la souffrance du corps feint de Lavinia. La figuration de la souffrance estelle prise en charge essentiellement par le costume, qui dès lors fait écran au corps? Ou au contraire le corps feint et le corps réel se confondent-ils au point que ce dernier fasse office de costume ? Quelle est la place du costume dans la relation qui s'instaure entre ces deux instances corporelles - le corps du comédien et le corps feint - et le spectateur?

Bien sûr le corps réel du comédien est toujours nécessairement engagé dans la figuration. Il s'agit d'examiner la nature de cet engagement ainsi que la fonction que l'on assigne au costume. Par ailleurs, le choix d'un type de costume nous renseigne sur la réaction que le metteur en scène entend activer chez le spectateur. Sollicite-t-il l'admiration ou au contraire la répulsion ? Les partis pris de mise en scène, nous allons le voir, divergent.

Mon étude portera principalement sur des mises en scène récentes, françaises et britanniques. Je m’appuierai donc sur les

${ }^{2}$ C'est notamment le cas de Eugene Waith, dans son article célèbre « The Metamorphosis of Violence in Titus Andronicus ", in Shakespeare Survey, $\mathrm{n}^{\circ}$ 10, (1957), p. 26-35. 
versions proposées par Bill Alexander (en 2003), Xavier Leret (en 2002), Lukàs Hemleb (en 2004), et Luc Bondy (en 2005). C'est à l'aune des mises en scène de Peter Brook (en 1955) et de Deborah Warner (en 1987) qu'il faudra les envisager. Ces deux versions ont en effet acquis un statut particulier dans l'histoire de la mise en scène, et sont souvent considérées comme des références notables, auxquelles renvoient la plupart des metteurs en scène contemporains.

En 1955, Peter Brook met en scène au Memorial Theatre de Stratford une version de Titus Andronicus qui fait date. Le succès de cette production redonne à la pièce droit de cité dans l'œuvre shakespearienne. Il s'agit de la première mise en scène véritablement signifiante de l'époque contemporaine, puisque la pièce n'avait été jouée en Angleterre, dans la première moitié du $\mathrm{xx}^{\mathrm{e}}$ siècle ${ }^{3}$, qu'une seule fois. Elle n'avait pas alors rencontré les faveurs du public. C'est en quelque sorte la version initiale, un cas d'école, à partir de laquelle la mise en scène contemporaine réfléchit. C'est d'ailleurs plus particulièrement à l'épisode du viol de Lavinia que l'on renvoie pour parler de sa mise en scène. Le traitement que Brook en fait est devenu emblématique et exemplaire.

La mise en scène de Brook se caractérise d'abord par une occultation presque systématique de la violence. Les corps morcelés ne sont jamais offerts directement à la vue, ils sont pudiquement dérobés par des linges. Quand il ne coupe pas le texte, Brook ménage donc une figuration euphémistique des données proposées par l'auteur. Le tissu lui sert à cacher mais aussi à signifier. Il est à la fois un procédé d'occultation et de présentation symbolique ou métaphorique. Il dissimule le corps, raconte le morcellement effroyable, et se substitue aux plaies.

Sans doute l'épisode du viol et de la mutilation de Lavinia est-il l'exemple le plus flagrant de ce procédé. La première partie de la scène, où l'on assiste aux suppliques vaines de Lavinia, semble avoir été jouée de manière réaliste. Les spectateurs voient d'abord la fille de Titus brutalement emmenée par ses violeurs hors de scène. Les spectateurs ne peuvent douter de l'horreur de ce qu'elle va vivre. En revanche, ce à quoi ils assistent par la suite, c'est à une représentation sublimée de la souffrance, dont la beauté les a, dit-on, fait gémir d'admiration. Antony

\footnotetext{
3 Mise en scène par Robert Atkins, en 1923, au Old Vic Theatre.
} 
Sher rapporte, dans Woza Shakespeare 4 , une anecdote sans doute révélatrice. Dans cet ouvrage qui retrace sous forme de journal ses recherches et celles de G. Doran pendant les répétitions du Titus mis en scène en 1994 en Afrique du Sud, Sher raconte une discussion qu'il a eue avec Janet Suzman - ayant joué elle-même Lavinia dans la mise en scène de Trevor Nun en 1972. L'actrice avait assisté à une représentation de la mise en scène de Brook et elle se rappelait la réaction du public au retour sur scène de Vivien Leigh, après l'épisode du viol. Le public avait alors laissé échapper un murmure d'admiration. Ce n'est pas la douleur représentée qui les avait fait réagir, comme l'imaginait Sher, mais la beauté de la vision qui leur était proposée. Les photos de cet épisode, devenu très célèbre, nous permettent de nous figurer un tant soit peu cette vision. Vivien Leigh ${ }^{5}$-Lavinia est ramenée sur scène par ses bourreaux. Ils la laissent debout, la tête pendante, les cheveux en désordre, le poing gauche dans la bouche. De ses poings et de sa bouche pendent des rubans écarlates, symbolisant ses mutilations.

Le rouge du tissu explicite l'analogie, son caractère vaporeux et flottant représente et poétise le flot de sang, les plaies qui coulent. Le caractère organique du corps est absent d'une telle représentation. Ce n'est pas sur son aspect profondément animal, faillible, fragile que l'on attire l'attention. Les blessures de Lavinia sont à proprement parler sublimées. Cette dernière devient davantage l'emblème de la souffrance qu'un corps effectivement mutilé et sanglant, présenté au regard des spectateurs. Le procédé employé par Brook pour signifier le sang et les plaies, c'est-à-dire le costume, ne propose pas une stricte analogie. Certes la couleur, la fluidité du tissu, servent d'élément comparatif, mais, par la même occasion, elles épurent l'acte représenté puisqu'elles le désincarnent de fait, le privent de sa matérialité. Le costume se substitue au corps réel de l'actrice, qui est caché ou déguisé, et au corps du personnage qu'il signifie. Il instaure un autre code de représentation, essentiellement non-réaliste, qui ne s'appuie plus sur le corps réel de l'acteur comme médium de figuration. Plus rien ne se joue dans le corps, que ce soit celui des personnages-acteurs ou celui

${ }^{4}$ Antony Sher et Gregory Doran, Woza Shakespeare, Titus Andronicus in South Africa, p. 217-218, Methuen Drama, 1996.

5 On peut renvoyer aux photos d'archives sur le site Internet de la RSC, rsc.org.uk., qui montrent cet épisode. Nous reprenons également en partie la description qu'en a faite Richard David dans son article pour le Shakespeare Survey, nº10 (1957), p. 27-38. 
des spectateurs, qui ne peuvent pas, comme s'en réjouissent certains critiques, avoir l'estomac retourné6. L'obscénité du corps blessé, des plaies qui coulent, est évacuée du champ de la représentation et peutêtre de la séance elle-même. Grâce à l'usage qu'il fait du costume, Brook leur a substitué une autre matière, qui n'évoque pas trop frontalement la dégradation organique potentielle. C'est sans doute la crainte de la relation obscène que l'on décèle dans les discours rassérénés des critiques de l'époque. Ils ont en effet su gré à Brook d'avoir pudiquement dissimulé les corps mutilés7. La répulsion fait place ici à l'admiration.

Dans son ouvrage Shakespeare's Violated Bodies, Stage and Screen Performances ${ }^{8}$, Pascale Aebisher raconte à quel point Vivien Leigh avait été mortifiée des choix de mise en scène, qui réduisaient considérablement son rôle et sa présence dans la pièce. Elle se vengeait, parait-il, en murmurant sur scène des injures à son mari. Elle avait été cantonnée, en quelque sorte, dans la posture d'icône muette et superbe de la souffrance, prisonnière de ce costume tragique et impénétrable.

En 1987, Deborah Warner propose au Swan Theatre une nouvelle mise en scène phare de la pièce9. Dans le dispositif scénographique élisabéthain du Swan Theatre, qui induit une participation physique plus réelle des spectateurs à la représentation, $\mathrm{D}$. Warner fait le choix de la déshistoricisation. Le plateau est pratiquement nu, les matériaux des quelques accessoires employés, comme le bois ou le métal d'une échelle, participent au parti pris transhistorique. Les costumes s'inscrivent dans cette perspective. Les matières simples et brutes utilisées évoquent la rudesse de l'antiquité, son caractère plus primitif que romain. Le costume de Lavinia composé d'une jupe et d'un haut à la coupe simple, est fait d'une toile orangée épaisse et légèrement gaufrée. Après le viol, ce costume est maculé de boue. Les manches, bouchonnées, sont également recouvertes de boue et figurent les mutilations. Cet usage de la boue sur le costume est particulièrement intéressant. Il donne à voir le hors-scène, le lieu épouvantable où le viol

\footnotetext{
${ }^{6}$ Cf. article de J. B. Boothroyed, in Punch du 24 août 1955.

${ }^{7}$ Je me réfère ici par exemple aux articles de Boothroyd, ibid. ou de Reese, dans le $\mathrm{n}^{\circ} 21$ de Shakespeare Quarterly de 1970.

${ }^{8}$ Cf. p. 42 et suivantes, Cambridge, C.U.P., 2005.

${ }^{9}$ Des photos de cette mise en scène sont consultables sur le site de la RSC, op. cit.
} 
a été commis. Dans l'acte II, Shakespeare met en place un réseau topique effroyable. Il superpose ainsi trois espaces redoutables: la forêt, cadre général de la chasse, le val reculé et enfin la fosse, dans laquelle sera jeté le corps de Bassianus et qui, par contamination, concentre les caractéristiques des deux autres lieux qui l'englobent et devient ainsi le comble de l'effrayant. Par ailleurs, une indétermination terrible est suggérée pendant une partie de la scène iii. On pense un temps que le même lieu, la fosse, servira de « recoin » secret, propice au viol, et de tombe à Bassianus. Chiron propose en effet de faire du tronc de Bassianus «[a] pillow to [their] lust » (II.iii.130). La simple présence de la boue sur le costume renvoie à ces espaces. Le costume et le texte entrent en résonance. La boue est alors à même de figurer l'abjection des bourreaux, le lieu immonde du viol. Comme la boue qui le constitue, et dont le costume garde des traces, c'est un lieu " nauséeux » et «informe »- sème selon Bataille, dans l'Histoire de l'Érotism $e^{10}$, de notre effroi face à la mort. Par métonymie le lieu du viol représente le viol lui-même.

En revanche, la mise en scène a peu recours au sang. On n'en trouve pratiquement pas de traces par exemple sur les moignons. Seul un mince filet de sang coule de la bouche de Lavinia alors que Marcus évoque, lorsqu'il la découvre, un "flot de sang » ${ }^{11}$. L'hyperbole employée par Marcus est figurée avec parcimonie. Deborah Warner retarde son apparition, ce qui redonne force et peut-être raison d'être au monologue de Marcus. Ce n'est véritablement qu'à ce moment-là que la représentation de Lavinia atteint son comble, et le public, qui a pourtant déjà assisté à ses tremblements spasmodiques d'oiseau blessé12, ne peut réprimer un soupir ému. Finalement, l'usage parcimonieux que Warner fait du sang rend l'effet recherché plus frappant. Le costume fait écho au texte sans le paraphraser de manière stricte : la boue renvoie au lieu du viol suggéré dans le texte sans le décrire davantage; le sang renvoie par exemple au monologue de

\footnotetext{
${ }^{10}$ Histoire de l'Érotisme, Euvres intégrales VIII, NRF, 1976. Cf. notamment p. 70 : « Nous n'avons pas de phobie plus grande que celle de ces matières mouvantes, fétides et tièdes où la vie fermente ignoblement. Les matières où grouillent les œufs, les germes et les vers ne nous serrent pas seulement, mais nous lèvent le cœur. La mort n'est pas réduite à l'amer anéantissement de l'être, de tout ce que je suis, qui attend d'être encore, dont le sens même, plutôt que d'être, est d'attendre d'être [...] c'est aussi le naufrage dans le nauséeux. »

${ }^{11}$ Il s'agit ici de la traduction de Jean-Pierre Richard pour la Pléiade, op. cit, II.iv.23.

12 J'emprunte ici, en la traduisant, la formule à Alan. C. Dessen, in Shakespeare in Performance : Titus Andronicus, Manchester U. Press, 1989, p. 66.
} 
Marcus sans l'illustrer simplement. La vision de Marcus reste subjective. Les traces du supplice que le costume conserve ne sont pas à envisager de manière strictement réaliste. Sans doute le costume devrait-il être davantage taché de sang, peut-être plus déchiré également, si l'on adoptait un tel point de vue. Mais les indices qu'il fournit, la sémiotique dans laquelle il engage le spectateur, suffisent à donner à voir l'horreur de ce que Lavinia a subi. Le costume acquiert une fonction narrative : chaque élément qui le constitue raconte ce qui s'est produit. Ce n'est pas seulement le corps souffrant qu'il donne à voir : il permet aux spectateurs de se figurer ce que le texte occulte, le hors-scène terrifiant.

En 2003, Bill Alexander met en scène la pièce au Main Theatre de la RSC à Stratford ${ }^{13}$. Plusieurs éléments de sa scénographie et des costumes renvoient à la Rome antique, mais il s'agit davantage d'évoquer une Rome conceptuelle que de représenter une période historique précise. Alexander met en scène une "romanitude », qui s'illustre dans les costumes, alliant des éléments romains, comme les toges à des pantalons ou à des $\mathrm{T}$-shirts plus contemporains. La robe bleue de Lavinia, dans la première partie de la pièce, participe de ce feuilletage temporel. Elle est théâtrale plus que romaine. Elle renvoie au statut social et à la fonction de Lavinia dans la pièce. C'est cette robe sobre et sage qu'elle arbore au moment de la terrible partie de chasse. Il s'agit d'une robe longue, qui recouvre les bras et les jambes, le col en $\mathrm{V}$ dégage assez peu la gorge. C'est ce costume, celui d'une Lavinia chaste, qui va être souillé et déchiré pour figurer l'outrage.

L'adjectif « réaliste » revient presque systématiquement sous la plume de Bill Alexander quand on lui demande d'évoquer les principaux épisodes de violence de la pièce. C'est le cas notamment du supplice de Lavinia. Alors que le viol a eu lieu hors de scène, on voit Lavinia, chancelante et comme secouée de convulsions, sortir d'une brèche faite dans le mur du fond : Lavinia sort d'un endroit effrayant et indéterminé, derrière la scène. Dans une lettre qu'il m'a adressée récemment, Bill Alexander décrit Lavinia ainsi : "The way she looked was realistic, not symbolic (no red ribbons or anything like that). Realistic bloody stumps, a face smeared with blood, her dress torn, muddy and soaked in blood ». Il fait allusion explicitement à la

\footnotetext{
13 Des photos de cette mise en scène sont consultables sur le site de la RSC, op.cit.
} 
méthode employée par Brook, et la rejette. On retrouve ici l'usage de la boue, comme chez Deborah Warner. En revanche c'est avec beaucoup plus de profusion que Bill Alexander emploie l'hémoglobine des chiens. Cette pléthore cruelle a d'ailleurs exaspéré certains critiques, comme la journaliste Kate Bessett qui, dans son article du 28 septembre 2003 de Independent on Sunday ${ }^{14}$, dit avoir eu du mal à croire en la violence exhibée. Elle s'est laissée envahir par des pensées intrusives, qui avaient à voir avec les techniques employées, les «trucages » de mise en scène, dont le costume participe ici. Le col de Lavinia, comme ses moignons, étaient en effet trempés de sang. La robe était déchirée au niveau des mains, et la blessure de la mutilation figurée réalistement par le maquillage et l'agencement du tissu. Les cheveux de l'actrice étaient emmêlés, et elle avait le visage meurtri de bleus. Le costume, assorti du maquillage, figurait explicitement ce qu'elle a subi. Ils participaient à créer l'illusion réaliste souhaitée par le metteur en scène. Le costume devait rendre possible l'assimilation du corps réel et du corps feint, et son emploi était assez proche de celui du costume de chair, qui couvre la nudité réelle d'un tissu mimétique, donnant l'illusion de la nudité.

Alexander fait du costume le support de l'identification. Le spectateur doit admettre que les taches de sang, la boue, représentent le réel et la souffrance du protagoniste. La sollicitation empathique, s'appuyant sur une figuration réaliste, se trouve inscrite dans ce dispositif didactique : il veut donner à voir, dans toute son horreur, les conséquences de la vengeance.

Lorsque Lukàs Hemleb met en scène la pièce en 2004 au CDN de Gennevilliers, il choisit également dans une certaine mesure la voie du réalisme. Il cherche à surprendre par l'illusion. Il me révélait en effet dans un entretien de décembre 2004 que ce qui l'intéressait, "c'est la fraction de seconde où l'on reçoit l'image sans distance ». " C'est trop facile, affirme-t-il, de dire que le théâtre ne peut pas rendre crédible le sang, alors on ne le montre pas ». Shakespeare est un poète de la matière, il faut donc rendre la matérialité du sang. La mise en scène doit en faire usage, parce qu'il renvoie à « des choses primitives et religieuses » et que «cette mythologie doit exister dans la tête du spectateur ». Le costume de Lavinia va donc intégrer fortement cette

${ }^{14}$ Article du 28 Septembre 2003. 
matière et rendre compte, de manière réaliste, de la souffrance du personnage.

L'actrice qui joue Lavinia porte dans cette mise en scène quatre costumes différents, le deuxième costume étant une version altérée du premier, faisant état du supplice subi. Dans la première partie de la pièce, Lavinia porte une robe blanche, dont le drapé et la toile épaisse peuvent évoquer Rome, mais cette robe est surmontée d'un haut marron aux manches bouffantes à partir du coude, et renvoie discrètement à la Renaissance. Les sandales et la coiffure sont romaines. Le costume est saillant et discret, le décolleté est peu prononcé, contrairement au costume largement décolleté de la lascive reine des Goths. Lavinia est mise en valeur sans ostentation. Ce costume a donc une fonction d'abord référentielle: préciser qui est Lavinia. On va voir par la suite que certains détails du costume relèvent du choix technique et vont permettre d'asseoir l'illusion. En effet, les manches bouffantes ne sont pas seulement esthétiques, elles permettent au «trucage » d'être efficace. Lukàs Hemleb m'a en effet confié avoir souvent été gêné par les proportions des bras de Lavinia après sa mutilation. Si l'on ferme les poings de l'actrice et qu'on les dissimule sous le tissu pour créer l'illusion des moignons, les bras se révèlent en réalité trop longs. Dans sa mise en scène, Lukàs Hemleb a décidé de cacher les avant-bras de l'actrice dans les manches et d'ajouter des prothèses figurant la mutilation. Ce sont elles qui sont visibles et qui donnent l'illusion des moignons, reproduisant ainsi les bonnes proportions. Le metteur en scène est donc très sensible aux détails, et il cherche précisément l'illusion réaliste. Le reste du costume parfait ce choix. Après le viol de Lavinia, Démétrius et Chiron la ramènent sur scène et la jettent sur le sol. Elle est recroquevillée, de dos, et le spectateur n'aperçoit pour le moment que ses épaules dénudées. Le public découvre véritablement Lavinia avec Marcus, dans la scène qui suit. Lorsqu'elle se retourne alors, on s'aperçoit que sa robe, descendue jusqu'au dessus de la poitrine, est tachée de sang à plusieurs endroits : au niveau du sexe, au niveau du cou, et l'intérieur des manches est maculée. Ce n'est que lorsque Marcus la priera de révéler le nom de ses bourreaux qu'un flot important de sang coulera de sa bouche et viendra souiller son visage. Dans cette mise en scène c'est donc essentiellement le sang sur le costume qui raconte le viol hors scène. Sa présence à la fois massive et retardée doit assurer l'effet, 
créer le choc du public. Outre l'usage important de l'hémoglobine des chiens sur le costume, la posture de l'actrice signifie la souffrance. Elle est à genoux, la tête doucement penchée sur le côté droit. Elle conservera cette posture, presque codée et esthétique, dans la scène suivante avec son père. Le geste délicat contraste avec la profusion du sang. L'image devient poétique. Si le costume crée le choc, il est ensuite adouci par l'image délicate, par la beauté du corps réel, en partie dénudé, et courbé dans une position codée de la souffrance. La compassion succède alors peut-être à l'horreur. Le corps réel de l'actrice est certes investi dans la représentation, mais il n'entre pas en concurrence avec le costume. Le costume dit le corps mutilé, l'horreur subie, dans un souci mimétique qui dissimule un temps - le temps bref du choc - le corps réel. Mais c'est le corps de l'actrice, séduisant malgré le supplice raconté, qui reste le médiateur de l'émotion qui suit.

La démarche adoptée par Xavier Leret ${ }^{15}$ est bien différente : il propose une version extrême de confusion du corps réel de l'actrice et du corps feint, l'inscrivant dans une sémiotique agressive et volontairement dérangeante. Dans l'espace claustrophobique du River Side Studio 3, les spectateurs ne peuvent échapper à la vision qui leur est imposée. Ce type d'espace suppose d'emblée un mode de participation plus physique des spectateurs, à portée de corps, si l'on peut dire, des acteurs. Les choix de mise en scène, et notamment ceux qui concernent le costume, renforcent la sollicitation viscérale du spectateur. Xavier Leret semble trouver ses sources d'inspiration à la fois dans le théâtre contemporain britannique que l'on a qualifié de " in yer face ", et dont les pièces se sont d'ailleurs beaucoup données dans de petites salles, et le film d'horreur. Le metteur en scène opère une série de brouillages spatiaux, de confusions, auxquels concourt le costume. Tout d'abord, le public et les acteurs partagent presque un même espace, et cet espace est terrible. C'est celui d'un abattoir ou d'une morgue, fait de carreaux blancs, froid et fonctionnel - on peut facilement laver les éclaboussures de sang. Au centre, un frigidaire qui s'ouvre à deux battants. Par ailleurs les costumes sont résolument contemporains, accentuant l'analogie entre le monde représenté et le monde réel du spectateur. L'implication des corps, et donc de celui de l'actrice qui joue Lavinia, le

15 On peut voir un montage d'extraits de la pièce, et plus particulièrement de cet épisode, sur le site www.xavierleret.com. 
choix de son costume, s'inscrivent dans cette perspective. Dans la première partie de la pièce, le costume renseigne d'abord sur son statut social et sur sa fonction dans l'intrigue, comme chez Bill Alexander, à cette exception près que les références sont contemporaines. Le costume de Lavinia est sage, on serait tenté de le qualifier de « bourgeois ». Elle porte un ensemble beige, composé d'une jupe à plis plats, au dessous du genou, et un chemisier classique. Une large écharpe de même couleur lui couvre les épaules. Elle a aux pieds des chaussures à talons plats et ses cheveux sont ramenés en arrière. Tout cela est marqué socialement et contraste avec le pantalon de cuir qu'arbore Tamora dans la scène de la forêt. La confrontation de Lavinia, avec Tamora et ses fils est d'une rare violence. Lavinia est jetée à terre, frappée contre les murs. Pour la faire taire, ce qu'il fera de manière au combien radicale par la suite, Chiron lui enfonce un doigt dans la bouche, figurant à la fois le viol et la mutilation de la langue. Quand elle est emmenée hors de scène, le spectateur a donc déjà assisté à une scène éprouvante. Ce n'est rien en comparaison de ce à quoi il va être confronté. Lorsqu'elle entre en scène, elle se présente d'abord de dos, appuyée contre le mur du fond. Elle a du mal à se soutenir et son costume est littéralement trempé de sang. Cette mise en scène en fait effectivement un usage massif. Sa bouche, appuyée contre le mur, laisse derrière elle une longue traînée de sang. Au moment où elle se retourne, les spectateurs constatent l'ampleur de son supplice. Sa jupe est déchirée, on aperçoit le corps dénudé de l'actrice, et le sang qui coule le long de ses jambes témoigne explicitement du viol. Xavier Leret fait le choix d'une hyper-représentation, et il gomme toutes les sources possibles de distance, toutes les formes d'esthétisation. Le costume exhibe le corps réel de l'actrice, il ne le supplée pas. Il n'est plus seulement mimétique, il met en danger l'acteur parce qu'il l'expose. L'usage du maquillage participe à cette impression de réel, renforcée par l'espace restreint de la salle. L'implication du corps réel de l'actrice gomme les frontières de la représentation. L'incarnation est totale et incontestable. Il s'agit donc d'une représentation qui se donne des allures de performance. Si le corps exposé est réel, le corps violé est bien encore celui de Lavinia. Si l'on amène le spectateur à croire qu'il a réellement en face de lui une femme violée, il n'en demeure pas moins qu'il suit le déroulement d'une intrigue. Le corps de l'acteur assume donc ici la fonction de costume. On peut envisager la nudité réelle de 
l'acteur comme un costume, à partir du moment où c'est au nom du personnage qu'il agit. Sa propre peau devient l'infime distance qui le sépare de son rôle. Mais cette pratique est fort troublante pour le spectateur, qui, pris dans un dispositif complexe, n'a peut-être plus le temps de se positionner en distance face à la représentation. Il est happé par le spectacle, et le metteur en scène cherche alors à susciter le malaise, la répulsion. La première scène, l'arrivée des Goths à Rome, simplement enroulés dans des couvertures, était donnée à voir à travers un écran, comme celui de la télévision. Alors que certaines actions violentes sont mises à distance par ce biais, le spectacle de Lavinia est au contraire on ne peut plus direct. Xavier Leret intègre donc la sollicitation viscérale dans un processus qui interroge le public sur son rapport à la violence, et à la violence envisagée comme spectacle.

Le costume participe ici du dispositif général de sollicitation viscérale qu'induit la mise en scène.

Lorsque Luc Bondy met en scène Viol, de Botho Strauss, la réécriture de la pièce de Shakespeare, il adopte une démarche analogue. Certes, la configuration des Ateliers Berthier est bien différente de celle du River Side Studio ${ }^{16}$, mais son utilisation du corps réel de la comédienne qui joue Lavinia est comparable. La scène de la forêt, qui précède le viol et la mutilation de Lavinia, reprend en les accentuant les données shakespeariennes : par exemple, Lavinia est attachée au corps de son mari assassiné, donnant à voir la réplique de Chiron qui parle de faire de Bassianus «un oreiller à leur luxure ». Luc Bondy décide de retarder le retour de Lavinia sur scène. Elle est encore dissimulée, en l'occurrence dans une poubelle, un immense container vert, lorsque Chiron et Demetrius se déchaînent en cruelles moqueries. Lorsque Lavinia sort de la poubelle, le choc est alors encore plus grand chez les spectateurs. Ils la découvrent presque entièrement nue, le corps et le visage maculés de sang, les poignets enveloppés de plastique. Dans un récent débat à la Comédie Française, le 23 février 2008, le dramaturge de la pièce, Daniel Loayza, revient sur les choix de cette mise en scène. Il souligne d'abord que l'actrice n'est pas totalement nue (elle porte en effet un soutien-gorge couleur chair) mais qu'elle doit donner

${ }^{16}$ La jauge, quoique moyenne, est malgré tout plus importante. La salle et le plateau sont plus vastes. 
l'impression aux spectateurs qu'elle l'est. Le costume doit ici se confondre avec le corps réel. Il explique aussi comment le maquillage a été très travaillé. Il fallait rendre précisément la matérialité du sang, l'aspect mouillé, l'éclat humide. Il s'agissait de figurer le plus précisément possible un corps souffrant. Le traitement du corps et du costume participait de cette recherche, d'autant plus que l'on donnait à voir un corps nu et rampant (c'est ainsi que Lavinia s'extrayait de la poubelle où l'avaient jetée ses bourreaux). La violence de la scène était d'ailleurs renforcée par le brusque éclairage de la salle. C'est en effet juste après ce choc visuel que l'entracte avait lieu. La scène semblait alors contaminer le quotidien du spectateur car ce dernier n'avait pas vraiment le temps de sortir de la représentation avant d'être brusquement ramené à la réalité. Ce qui importait, selon Daniel Loayza, ce n'était pas seulement que les spectateurs voient ce corps humilié. La nudité prenait un sens plus déterminant encore dans la scène suivante : Titus y découvrait sa fille dans cet état lamentable. Ce que l'on voulait donner à voir aux spectateurs, c'est un père confronté à une telle vision.

La pièce de Botho Strauss fait alterner des scènes contemporaines et des scènes renvoyant directement à la pièce de Shakespeare. Le texte produit donc de lui-même un commentaire métathéâtral sur notre rapport à la violence, particulièrement perceptible dans la scène qui représente l'interview d'un metteur en scène caricatural. Encore une fois, on voit que le choc induit par la confusion du corps réel et du corps feint, ici par l'emploi d'un costume si discret qu'il exhibe surtout le corps du comédien, participe à un dispositif scénique qui interroge la position du spectateur face à la représentation de la violence.

Que le costume de théâtre puisse figurer le corps, rien d'étonnant. On connaît de longue date l'usage du costume de chair, qui dit la nudité sans exposer le corps. La mise en scène contemporaine investit la nudité réelle du comédien comme espace de représentation. Cette nudité réelle brouille les frontières, même s'il ne s'agit pas de porter de fait atteinte à l'intégrité du corps du comédien, de le faire souffrir réellement en scène. L'existence du body art complique sans doute encore davantage le problème, car, même si les mises en scène que j'ai 
étudiées ne relèvent pas de ce type de performance, elles font partie de l'horizon d'attente du spectateur, qui sait qu'elles sont un possible du théâtre contemporain.

Le rôle de Lavinia exacerbe la fonction même du costume, l'interroge et permet d'en cerner les limites. Le costume est en effet à la fois un élément du décor et du corps du comédien, l'interstice entre le corps joué et le corps réel qu'il protège ou expose. Aucune des mises en scène que j'ai analysées ne renonce à un usage référentiel - le costume précise toujours à qui l'on a à faire, et les lectures initiales du personnage sont somme toute assez comparables. C'est donc plutôt en fonction de l'effet recherché, de la nature du choc à produire chez le spectateur, qu'il faut réfléchir et spécifier ces mises en scène. Quelle est la part de répulsion, de compassion, d'admiration que l'on suscite ? Le rôle de Lavinia suppose une relation à l'obscène que les metteurs en scène choisissent d'activer ou au contraire d'occulter, d'ajourner dans l'imaginaire du spectateur. Ce que craint finalement le spectateur, ce qui l'effraie le plus, c'est peut-être cette possible rencontre obscène. Le traitement du costume rend alors tangible cette rencontre ou la sublime pour en proposer une version désincarnée. Il escamote le corps ou confronte le spectateur à sa matérialité réelle et dérangeante.

Isabelle BEUCHER

Université de Paris X 